PoS $\quad$ PROCEEDINGS

\title{
Light scalar mesons in 2+1 flavor full QCD *
}

\author{
$\chi$ QCD Collaboration: Terrence Draper $^{\dagger a}$, Takumi Doi ${ }^{a}$, Keh-Fei Liu ${ }^{a}$, Devdatta \\ Mankame $^{a}$, Nilmani Mathur ${ }^{b}$, Xiangfei Meng $^{a, c}$ \\ ${ }^{a}$ Department of Physics and Astronomy, University of Kentucky, Lexington, KY 40506, USA \\ ${ }^{b}$ Department of Theoretical Physics, Tata Institute of Fundamental Research, Mumbai 40005, \\ India \\ ${ }^{c}$ College of Physics, Nankai University, Tianjin 300071, P.R. China \\ E-mail: draperepa.uky.edu
}

\begin{abstract}
We study the $a_{0}$ and $K_{0}^{*}$ light scalar mesons in $2+1$ flavor full QCD. Particular attention is paid to fitting excited states, with an eye toward determining whether scattering states are revealed. An ultimate goal will be to see how dynamical quarks affect the picture outlined with an earlier quenched study using overlap fermions, namely, that it is the $a_{0}(1450)$, not the $a_{0}(980)$, which is the lowest $\bar{q} q$ isovector scalar state.
\end{abstract}

The XXVI International Symposium on Lattice Field Theory

July $14-19,2008$

Williamsburg, Virginia, USA

${ }^{*}$ This research is supported in part by the U.S. Department of Energy under grant DE-FG05-84ER40154. The research of N.M. is supported in part by DST-SR/S2/RJN-19/2007 (India). We thank CP-PACS/JLQCD for the use of their dynamical configurations. We thank S. Prelovsek and A. Hasenfratz for important and interesting discussions.

†Speaker. 


\section{Motivation}

The light scalar mesons are not unambiguously identified in terms of quark content and their $S U(3)_{f}$ classification. There are more experimental candidates for the $\bar{q} q$ nonet than can be accommodated in the simple quark model. Fig. 1 shows the current experimentally known-scalar, and other low-lying, mesons.

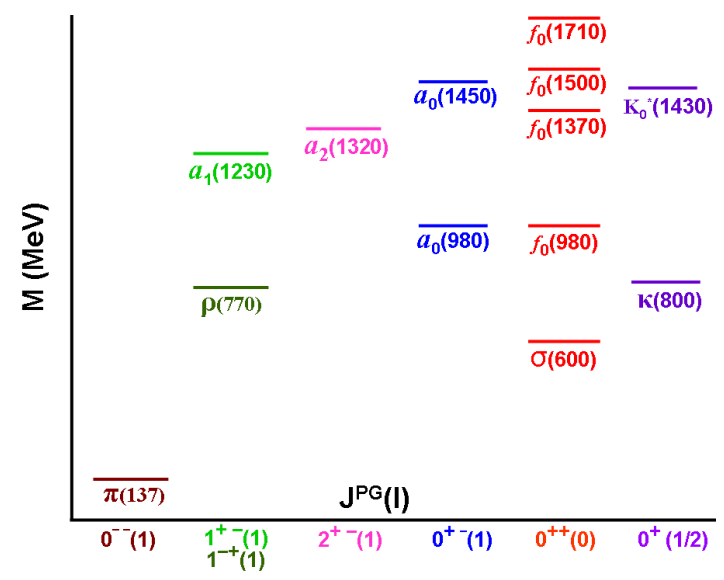

Figure 1: Spectrum of low-lying mesons.

An attractive classification of light scalar mesons emerges [1] which resolves many puzzles in the ordering of states and size of branching ratios. This is indicated in Fig. $2:$ a) a tetraquark nonet, including the $\sigma(600), a_{0}(980)$ and $f_{0}(980)$, below $1 \mathrm{GeV}$, and b) a $\bar{q} q$ nonet, including the $a_{0}(1450)$ and $K_{0}^{*}(1430)$, and an almost pure glueball, $f_{0}(1710)$, above $1 \mathrm{GeV}$.

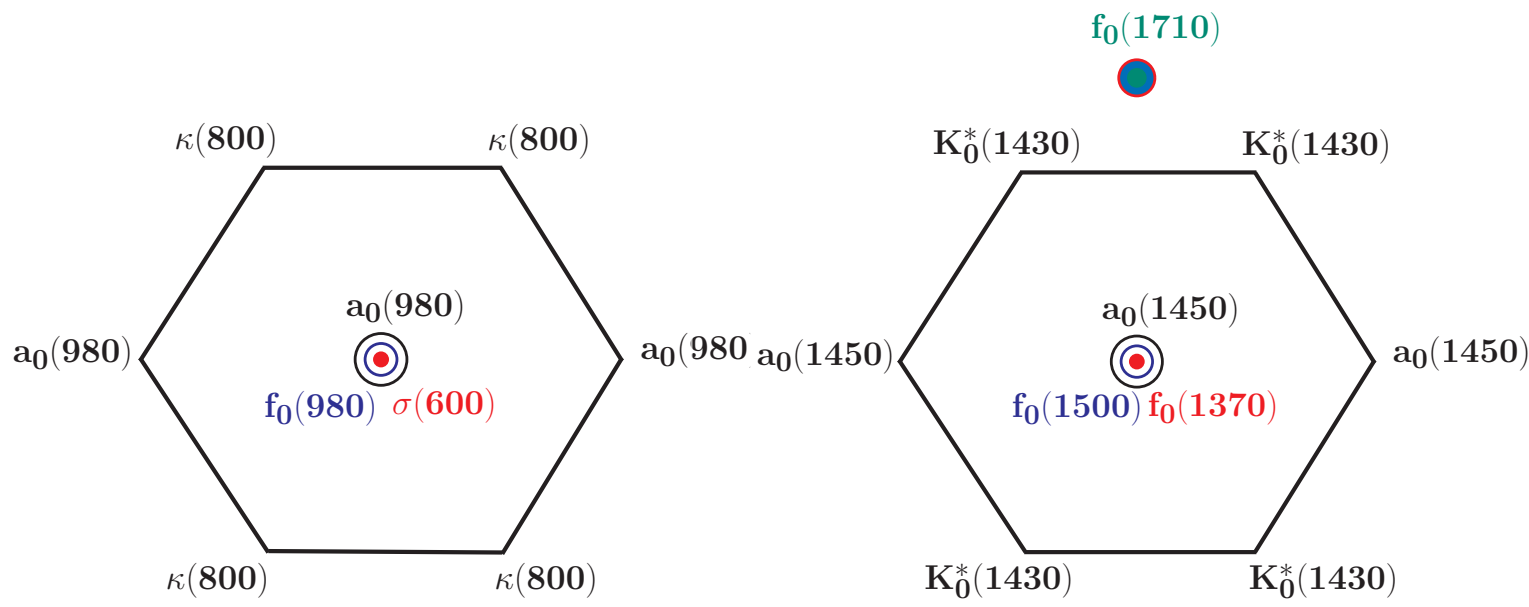

Figure 2: Proposed classification of light scalar mesons.

Our quenched calculation with overlap fermions [2] offered support of this view in providing evidence that the $\sigma(600)$ is a four-quark state. We also found evidence [2] that the lowest lying isovector scalar $\bar{q} q$ state is the $a_{0}(1450)$, not the $a_{0}(980)$. 
Fig. 3 shows the masses of the $a_{0}$ and $a_{1}$ as a function of quark mass. The $a_{0}$ has little dependence on mass, especially below the strange quark mass region, and extrapolates (from quite close to the chiral limit with pion mass as low as $180 \mathrm{MeV}$ ) to a mass of $1.42(13) \mathrm{GeV}$. Ghost would-be $\pi \eta^{\prime}$ states, a quenched artifact, were identified and handled by the fit, but no evidence of $a_{0}(980)$ was seen in this channel. Indeed it was excluded with a squared spectral weight less than 0.015 that of the $a_{0}(1450)$. This may account for why the $K_{0}^{*}(1430)$ and $a_{0}(1450)$ have approximately equal masses experimentally even though the former has a strange quark. This counters the conventional quark-counting rule; however, the constituent quark model may have limitations for light hadrons where chiral symmetry plays an important role.

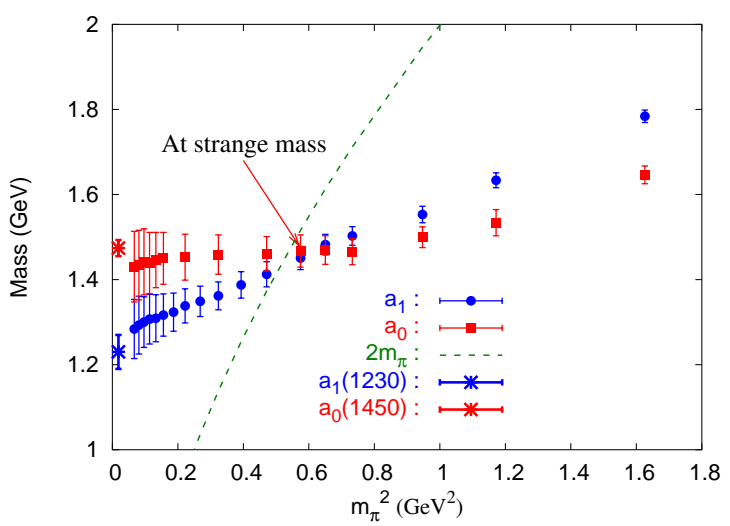

Figure 3: $a_{0}$ and $a_{1}$ masses are plotted as a function of $m_{\pi}^{2}$. Also shown is the two pion mass (dashed line) which becomes lower than the $a_{0}$ around the strange quark mass region.

The proposed picture, however intriguing, is nevertheless tentative. Early two-flavor dynamical simulations $[3,4]$ reported values at or above $1.5 \mathrm{GeV}$, but later two-flavor dynamical calculations $[6,7,8]$ obtained a ground state near $1 \mathrm{GeV}$. But is this the $a_{0}$ or $\pi \eta_{2}^{\prime}$ ? Dynamical simulations must identify both the $a_{0}$ and scattering state(s) before the issue is settled.

\section{Lattice Details}

We use the 2+1 flavor full QCD configurations provided by CP-PACS+JLQCD [9]. These have renormalization-group improved gauge action and non-perturbatively $O(a)$-improved clover quark action.

The lattice size is $16^{3} \times 32$ with lattice spacing $a=0.12 \mathrm{fm}$. The light sea quark masses have $\kappa=0.13760,0.13800,0.13825$, for which $m_{P S}(L L) / m_{V}(L L)=0.71,0.66,0.62$, with pion masses $0.84,0.70$, and $0.61 \mathrm{GeV}$. The strange sea quark mass has $\kappa_{S}=0.13760$. We use valence quark action and masses which match the sea quark (i.e. no partial quenching).

We compute local-local two-point correlation functions with very high statistics; for each of 800 configurations, we use 32 different delta-function sources (requiring 32 different valence quark matrix inversions) well separated in space and time.

We use the Sequential Empirical Bayes (SEB) method [10], a constrained-curve fitting algorithm, to fit the ground and some excited states. 


\section{Effective Masses and Fits}

We look first at the heaviest quark mass for which both strange and up/down quark masses (for both valence and sea) are equal at $\kappa_{S}=\kappa=0.13760\left(m_{\pi}=0.84 \mathrm{GeV}\right)$.

Fig. 4 (left) shows the effective mass plot for the $a_{0}$ (which is also the $K_{0}^{*}$ since these quark masses are degenerate). Fig. 4 (right) shows the $\chi^{2} /$ dof for various one-state (single-cosh) fits for the interval $[t, 16]$. We get good one-state fits over plateaus as long as $[5,16]$. Given a starting interval, the automated SEB method [11] adds earlier and earlier time slices, and determines when new terms need be added to the fit model.
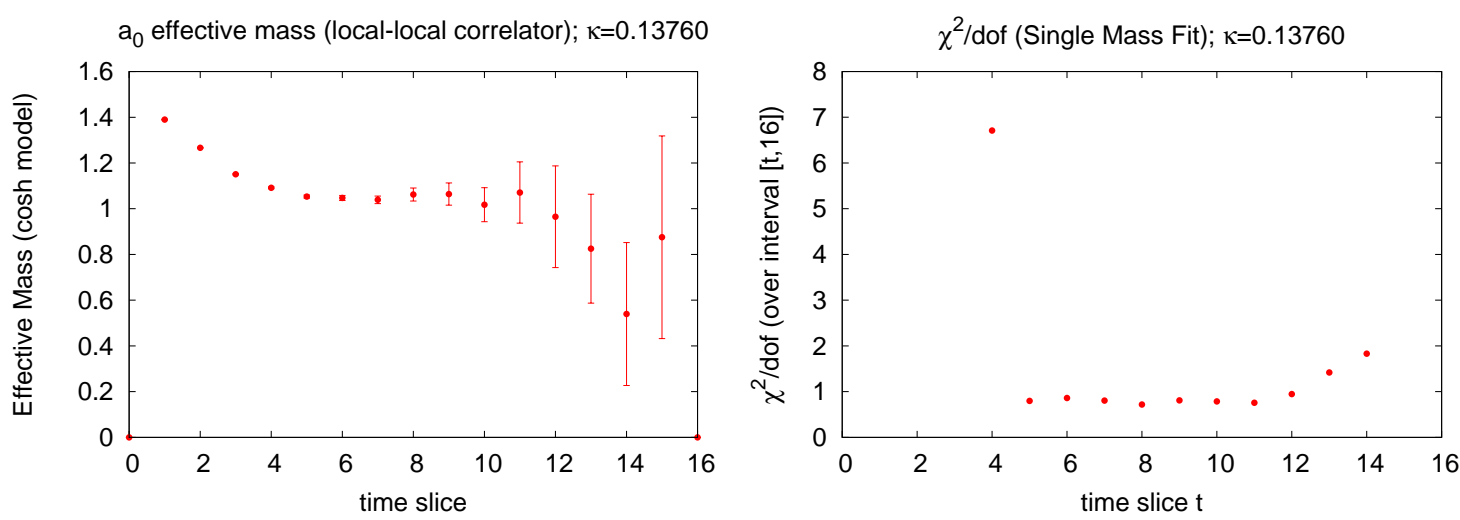

Figure 4: Left: Effective mass plot for the isovector scalar channel with both strange and up/down quark masses (for both valence and sea) equal at $\kappa=\kappa_{S}=0.13760\left(m_{\pi}=0.84 \mathrm{GeV}\right)$. All effective mass plots are in accord with a cosh, not exponential, model; that is, for a correlation function (with periodic boundary conditions) which asymptotically is saturated with a single mass term, the effective mass plot will be a plateau right up to the middle of the lattice. Right: $\chi^{2} / \operatorname{dof}$ for one-state fits over the intervals $[t, 16]$.

Final single or multi-state fits give consistent results for the ground-state mass; we obtain $1.04(1)$, i.e. $1.70(2) \mathrm{GeV}$, and a first-excited state much higher. The isovector scalar interpolating operator can excite $\pi \eta$ scattering states (as well as $\pi \eta^{\prime}$ and $K \bar{K}$ states). If $\pi$ and $\eta$ propagate in the same direction in time, then they give a contribution with energy $E=m_{\eta}+m_{\pi}+E_{\text {int }}$. At this quark mass, the scattering state $\pi \eta$ has an energy which is presumably close to that of the $a_{0}$ (since $\left.2 m_{\pi}=1.68 \mathrm{GeV}\right)$. In this case, the SEB method cannot resolve the two energies with our current statistics.

Next, we consider the case where the strange quark mass (for sea and valence) remains at $\kappa_{S}=0.13760\left(m_{\pi}=0.84 \mathrm{GeV}\right)$, but the light quark mass (for sea and valence) has $\kappa=0.13825$ $\left(m_{\pi}=0.61 \mathrm{GeV}\right.$ ). Fig. 5 (left) shows the effective mass plot. Fig. 5 (right) shows the $\chi^{2} /$ dof for various one-state (single-cosh) fits for the interval $[t, 16]$. Again we get low $\chi^{2} /$ dof for a wide range of values of $t$; that is, there is a long plateau.

Multi-state or single-state fits give consistent results for the ground-state mass of 0.83(2), i.e. $1.35(3) \mathrm{GeV}$. The first-excited state is much higher. Using the Gell-Mann-Okubo mass formula $3 m_{\eta}^{2}=4 m_{K}^{2}-m_{\pi}^{2}$ and the measured kaon mass of $0.68 \mathrm{GeV}$ to estimate the mass of the $\eta$ as $m_{\eta} \approx 0.70 \mathrm{GeV}$, we expect the $\pi \eta$ scattering state to have an energy near $1.31 \mathrm{GeV}$ which is close to the measured value of the ground state energy. 

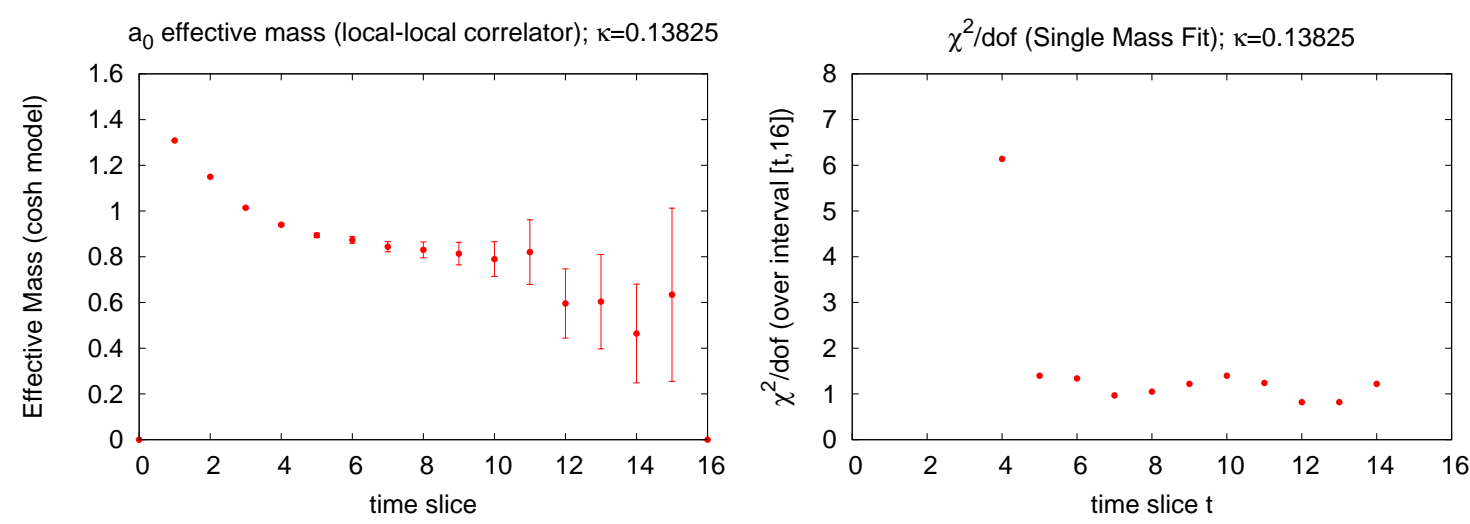

Figure 5: Same as for Fig. 4 except that while the strange quark mass (for sea and valence) remains at $\kappa_{S}=0.13760\left(m_{\pi}=0.84 \mathrm{GeV}\right)$, the light quark mass (for sea and valence) has $\kappa=0.13825\left(m_{\pi}=0.61 \mathrm{GeV}\right)$.

The dip in the effective mass plot at large Euclidean time hints of evidence of a peculiar effect due to a behavior of scattering states on a lattice with periodic boundary conditions in time. If the $\pi$ and $\eta$ propagate in opposite directions in time, they make a contribution with energy $\Delta m=m_{\eta}-m_{\pi}$ [5]. Indeed an artificially-constructed correlation function with such a "wraparound" contribution can agree with the data. The dip is not statistically significant however, and unfortunately, further doubling the statistics did not help. We cannot confirm this effect with our data, but it must be kept in mind in all future simulations. Rather than regard this as another contamination to isolate and control in an effort to extract the $a_{0}$ mass, one should take the optimistic view and regard this as a fascinating opportunity to measure the $\eta$ mass!

In an attempt to better isolate the $a_{0}$ in the correlator by eliminating this wrap-around effect, we recomputed the propagators with Dirichlet (fixed) boundary conditions in time. Unfortunately, our statistics are poor at large Euclidean times, and we are unable to get an improved fit.
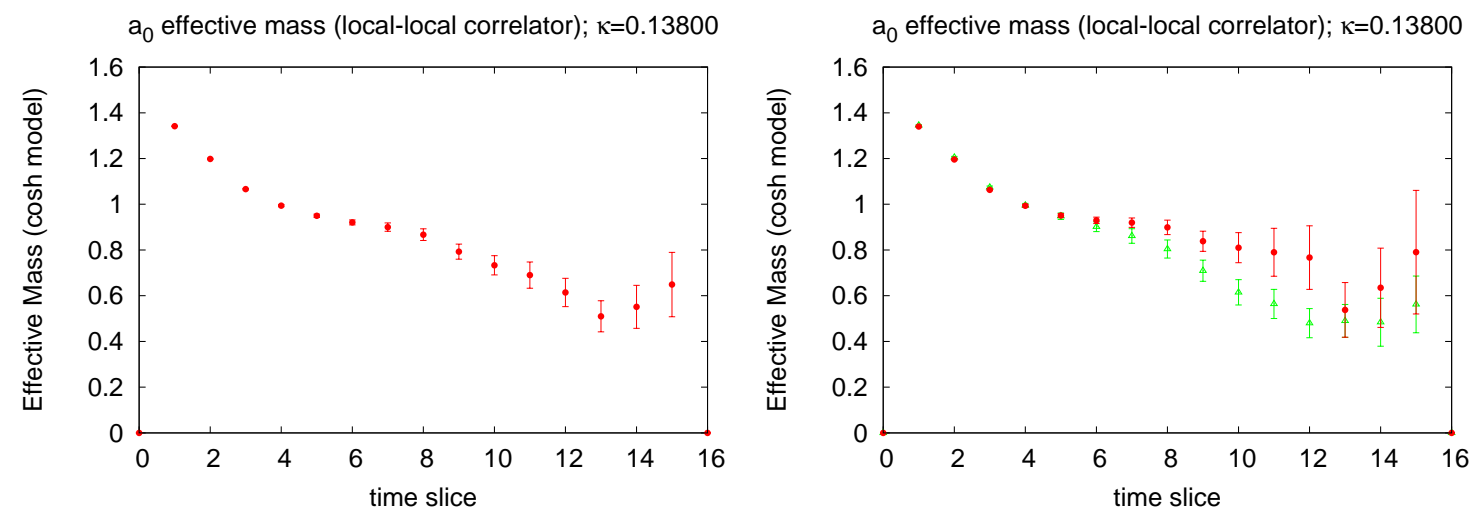

Figure 6: Left: Same as for Fig. 4 (left) except that while the strange quark mass (for sea and valence) remains at $\kappa_{S}=0.13760\left(m_{\pi}=0.84 \mathrm{GeV}\right)$, the light quark mass (for sea and valence) has $\kappa=0.13800$ $\left(m_{\pi}=0.61 \mathrm{GeV}\right)$. Right: The effective mass plot from the second trajectory of 230 configurations (green triangles) and that of the remaining two trajectories combined (red dots). 
The case where the strange quark mass (for sea and valence) remains at $\kappa_{S}=0.13760\left(m_{\pi}=\right.$ $0.84 \mathrm{GeV})$ and the light quark mass is intermediate at $\kappa=0.13800\left(m_{\pi}=0.70 \mathrm{GeV}\right)$ is most peculiar. Fig. 6 (left) shows the effective mass plot. In contrast to the previous two cases, here there is no stable plateau, and the error bars are smaller than expected. We cannot make a fit in this case.

The dynamical gauge configurations were created from three trajectories. Fig. 6 (right) shows the effective mass plot from the second trajectory of 230 configurations (green triangles) and that of the remaining two trajectories combined (red dots). The latter resembles those of the other masses, but the former is peculiar with relatively small error bars and lack of a plateau. This behavior of having one trajectory giving disparate results is not replicated in other channels.

In summary, we are only able to obtain ground state fits in the isovector scalar channel for two of the three light quark masses studied. Although we don't attempt a formal chiral extrapolation with only two points at rather large quark mass (with $m_{\pi}=0.84 \mathrm{GeV}$ and $0.61 \mathrm{GeV}$ ), the downward trend suggests a ground state mass near $1 \mathrm{GeV}$, rather than near $1.5 \mathrm{GeV}$, in this channel. At face value, this suggests that the lowest lying isovector scalar $\bar{q} q$ state is the $a_{0}(980)$, not the $a_{0}(1450)$. This agrees with that of other recent dynamical quark simulations $[6,7,8]$ and at first glance appears to be at odds with the picture painted with a quenched overlap calculation at low quark mass [2]. But we cannot jump to this conclusion because the measured ground state energies are so close to expected $\pi \eta$ scattering states. Dynamical simulations must identify both the $a_{0}$ and scattering state(s) before the issue is settled.

The use of smeared sources would alter the proportion of $\bar{q} q$ and scattering states, allowing SEB or a variational calculation to resolve the states. We might also expect a resolution with a new set of $2+1$ flavor dynamical gauge configurations at lighter quark masses.

\section{Hybrid Boundary Conditions}

For our quark masses, the kinematics are such that we expect there to be a $\pi \eta$ scattering state near the measured ground state in the isovector scalar channel. To reveal this, we recomputed the valence quark propagators with hybrid boundary conditions (HBC) [11], using the same configurations. In our version of $\mathrm{HBC}$, we use anti-periodic boundary conditions in space for the valence quarks, while the dynamical quarks still have periodic boundary conditions. Although the valence quarks are given non-zero momentum by the interpolating fields, a $\bar{q} q$ ground state will still have zero momentum. On the other hand, in the scattering state each meson must have non-zero momentum (since the lowest Fock component has one valence and one sea quark). Thus, HBC raise the energy of the scattering states while leaving that of $\bar{q} q$ unchanged, potentially allowing SEB to resolve the states. (A caveat is that with mixed boundary conditions (valence versus sea), ghost states may complicate matters.)

Using $\mathrm{HBC}$ for $\kappa_{S}=0.13760$ and $\kappa=0.13825$, we measure the energy of the lowest isovector scalar state to be $1.50(3) \mathrm{GeV}$. This is higher than the $1.35(3) \mathrm{GeV}$ measured with periodic boundary conditions (PBC). This strongly suggests that the measured PBC ground state energy (GSE) is that of a scattering state, and casts doubt that the $a_{0}$ mass extrapolates to a value near $1 \mathrm{GeV}$ in the chiral limit. However, this HBC GSE does not exceed the PBC GSE by the expected momentumdependent amount, if both are scattering states. Rather, the measured HBC GSE is less, and could be that of the $a_{0}$. 
We propose the following scenario which is consistent with our (limited) observations: at our lowest light quark mass the PBC GSE of $1.35(3) \mathrm{GeV}$ is that of a scattering state, with the singleparticle $\left(a_{0}\right)$ state lying higher. As HBC are imposed, the scattering state energy increases, leapfrogging that of the single-particle state which does not change much, leaving the single particle state exposed as the (new) ground state at $1.50(3) \mathrm{GeV}$. Furthermore, at higher light quark mass (degenerate with the strange), we observe that the GSE does not change much from PBC $(1.70(2) \mathrm{GeV})$ to HBC $(1.69(2) \mathrm{GeV})$, suggesting that the ground state in this case is a single-particle $\left(a_{0}\right)$ state. In addition, although a naive chiral extrapolation for $\mathrm{PBC}$ would give an extrapolated value near $1 \mathrm{GeV}$, this extrapolation should not be done, as it mixes single-particle and scattering states! The HBC GSE chiral extrapolation is much less steep than for the PBC GSE, with an extrapolated value much higher than $1 \mathrm{GeV}$.

Unfortunately, our evidence for such a scenario is incomplete. With the current data set, the statistics are not sufficient for SEB to convincingly extract excited states. We need to measure these to verify that we see HBC raise energies by the expected amounts. Then we can unambiguously identify and distinguish scattering states from single-particle states. Until this is resolved, we urge the community to keep an open mind about whether or not the $a_{0}(980)$ is a $q \bar{q}$ state.

Scalar mesons are an increasingly rich, fascinating, and frustrating forum.

\section{References}

[1] K.F. Liu, Pattern of Light Scalar Masses, Prog. Theor. Phys. Suppl. 168: 160-167, 2007; K.F. Liu, Challenges of Lattice Calculation of Scalar Mesons, arXiv:0805.3364.

[2] N. Mathur et al., Scalar Mesons $a_{0}(1450)$ and $\sigma(600)$ from Lattice QCD, Phys. Rev. D76 (2007) 114505 .

[3] T. Kunihiro et al. (SCALAR Collaboration), Scalar Mesons in Lattice QCD, Phys. Rev. D70 (2004) 034504.

[4] S. Prelovsek et al., Scalar meson in dynamical and partially quenched two-flavor QCD: lattice results and chiral loops, Phys. Rev. D70 (2004) 094503.

[5] We thank S. Prelovsek for alerting us to this possibility.

[6] C. McNeile, C. Michael, Properties of light scalar mesons from lattice QCD, Phys. Rev. D74 (2006) 014508 .

[7] R. Frigori et al., Dynamical Chirally Improved Quarks: First Results for Hadron Masses, PoS(LAT2007) 114.

[8] K. Hashimoto, T. Izubuchi (RBC Collaboration), $\eta^{\prime}$ Meson From Two Flavor Dynamical Domain Wall Fermions, Prog. Theor. Phys. 119 (2008) 599-641.

[9] CP-PACS/JLQCD Collaborations: T. Ishikawa, et al., PoS (LAT2006) 181; Phys. Rev. D78, 011502(R) (2008).

[10] Y. Chen et al., The Sequential Empirical Bayes Method: An Adaptive Constrained-Curve Fitting Algorithm for Lattice QCD, arXiv:hep-lat/0405001.

[11] N. Ishii et al., Penta-quark baryon in anisotropic lattice QCD, Phys. Rev. D71 (2005) 034001. 